\title{
Energy and Exergy Efficiency Analysis of Fluid Flow and Heat Transfer in Sinter Vertical Cooler
}

\author{
Zude Cheng ${ }^{1}$, Haitao Wang ${ }^{1,2} \mathbb{D}$, Junsheng Feng ${ }^{1,2, *}$, Yongfang Xia ${ }^{1,2}$ and Hui Dong ${ }^{3}$ \\ 1 School of Environment and Energy Engineering, Anhui Jianzhu University, Hefei 230601, China; \\ czd@ahjzu.edu.cn (Z.C.); wht@ahjzu.edu.cn (H.W.); xiayf@ahjzu.edu.cn (Y.X.) \\ 2 Advanced Technology Institute of Green Building Research of Anhui Province, Anhui Jianzhu University, \\ Hefei 230601, China \\ 3 School of Metallurgy, Northeastern University, Shenyang 110819, China; dongh@smm.neu.edu.cn \\ * Correspondence: fjsheng@ahjzu.edu.cn; Tel.: +86-551-6382-8252
}

Citation: Cheng, Z.; Wang, H.; Feng, J.; Xia, Y.; Dong, H. Energy and Exergy Efficiency Analysis of Fluid Flow and Heat Transfer in Sinter Vertical Cooler. Energies 2021, 14, 4522 https://doi.org/10.3390/en14154522

Academic Editor: Marcin Kamiński

Received: 5 July 2021

Accepted: 23 July 2021

Published: 27 July 2021

Publisher's Note: MDPI stays neutral with regard to jurisdictional claims in published maps and institutional affiliations.

Copyright: () 2021 by the authors. Licensee MDPI, Basel, Switzerland. This article is an open access article distributed under the terms and conditions of the Creative Commons Attribution (CC BY) license (https:// creativecommons.org/licenses/by/ $4.0 /)$
Abstract: In order to fully understand the energy and exergy transfer processes in sinter vertical coolers, a simulation model of the fluid flow and heat transfer in a vertical cooler was established, and energy and exergy efficiency analyses of the gas-solid heat transfer in a vertical cooler were conducted in detail. Based on the calculation method of the whole working condition, the suitable operational parameters of the vertical cooler were obtained by setting the net exergy efficiency in the vertical cooler as the indicator function. The results show that both the quantity of sinter waste heat recovery (SWHR) and energy efficiency increased as the air flow rate (AFR) increased, and they decreased as the air inlet temperature (AIT) increased. The increase in the sinter inlet temperature (SIT) resulted in an increase in the quantity of SWHR and a decrease in energy efficiency. The air net exergy had the maximum value as the AFR increased, and it only increased monotonically as the SIT and AIT increased. The net exergy efficiency reached the maximum value as the AFR and AIT increased, and the increase in the SIT only resulted in a decrease in the net exergy efficiency. When the sinter annual production of a $360 \mathrm{~m}^{2}$ sintering machine was taken as the processing capacity of the vertical cooler, the suitable operational parameters of the vertical cooler were $190 \mathrm{~kg} / \mathrm{s}$ for the AFR, and $353 \mathrm{~K}$ for the AIT.

Keywords: sinter; vertical cooler; gas-solid heat transfer; energy efficiency; exergy efficiency

\section{Introduction}

The sinter waste heat resource accounts for about $8 \%$ of the total waste heat resources of iron and steel enterprises in China, which is one of the resources producing large amounts of waste heat with the greatest development potential [1-4]. Sinter annular coolers and vertical coolers are two main types of equipment for sinter waste heat recovery (SWHR), and annular coolers have been widely used in the production of SWHR. Due to the characteristics of the structural style and SWHR in annular coolers, the recycled air temperature at the outlet of annular coolers is lower, as well as the utilization ratio of SWHR for cogeneration [5]. Vertical coolers, proposed for overcoming the disadvantages of existing annular coolers, are a type of highly efficient equipment for SWHR, and both the air temperature at the outlet of a vertical tank at above $450{ }^{\circ} \mathrm{C}$ and the utilization ratio of SWHR in a vertical cooler at above $75 \%$ are greater than those in annular coolers [6]. At present, vertical coolers for SWHR are still in the laboratory research stage, and detailed research of the flow and heat transfer in vertical coolers is quite important to analyze the theoretical feasibility of vertical coolers. The gas flow in the bed layer directly affects the gas flow resistance in vertical coolers and thus affects the power consumption of air blowers, while the gas-solid heat transfer in the bed layer directly affects the quantity and quality of SWHR in vertical coolers and thus affects the efficiencies of waste heat recovery and utilization. Therefore, detailed investigations of the flow and heat transfer in vertical 
coolers have an important significance for improving the recovery rate of sinter waste heat and optimizing the operational parameters of vertical coolers.

Most studies on the flow and heat transfer in the sinter bed layer were mainly conducted in annular coolers in recent years. Zhang et al. [7] developed flow and heat transfer models by correcting the momentum source term and local non-thermodynamic equilibrium double energy equations, and the influence factors and rules of the flow and heat transfer in annular coolers were analyzed in detail, while the optimal parameters of annular coolers were determined with the purpose of improving the waste heat utilization. Liu et al. [8,9] performed gas-solid heat transfer analysis in the sinter bed layer of an annular cooler, and the operational parametric effects on the quantity and quality of SWHR, as well as the equivalent annual operational cost, were investigated in detail. Tian et al. [10] simulated the flow and heat transfer processes of a layer loaded sinter cooler in order to increase the amount of SWHR and designed an orthogonal optimization experiment to analyze the effects of the designed parameters. Feng et al. [11] applied constructal theory to perform parameter optimization in an annular cooler, and the optimal cross-sectional shape of the bed layer was determined based on the maximum exergy output of SWHR. Zhang et al. [12,13] utilized local non-thermodynamic equilibrium double energy equations to investigate the main influence factors and influence laws of SWHR in annular coolers, and the effective method for improving SWHR was provided. In addition, Zhang et al. $[14,15]$ also performed exergy transfer analysis in the sinter bed layer of an annular cooler and analyzed the thermal parametric effects on the temperature exergy and pressure exergy in the heat transfer process. Tian et al. [16,17] established a simulation model of three-dimensional flow and heat transfer in an annular cooler and optimized the operating parameters of a ring annular cooler by taking the sinter outlet temperature and exergy of SWHR as the objective function. Some other relevant studies were also reported in the literature $[18,19]$.

In essence, a sinter annular cooler is a cross-convection fixed bed, and most of the above heat transfer models established in annular coolers are for unsteady-state heat transfer, which means that the particle movement in the bed layer is ignored, and the bed layer is treated as a stationary bed. Compared with an annular cooler, a vertical cooler is a type of counter-flow moving bed, in which the heat transfer process is a steady-state condition. Due to the complexity of the flow and heat transfer in vertical coolers, correlational studies for vertical coolers were mainly based on experimental research [20-22], while few numerical studies in vertical coolers were conducted, and thermal parameter optimization of vertical coolers as the critical issue was rarely considered and mentioned, which resulted in the parameter design of vertical coolers being based more on experience and a lack of theoretical guidance. In addition, the objective functions of parameter optimization in annular coolers mentioned above were mainly the quantity of SWHR and the outlet exergy of the heat carrier, which could not accurately reflect the relationship between system inputs and outputs. For all we know, energy and exergy efficiency analysis in vertical coolers was not carried out, and optimization analysis of the operational parameters in vertical coolers was also not performed by using the analysis method of exergy efficiency.

In conclusion, it is necessary to perform energy and exergy efficiency analysis of the sinter cooling process to improve the efficiencies of sinter waste heat recovery and utilization in vertical coolers. Therefore, in the present work, the specific expressions of energy and exergy efficiency in a vertical cooler were derived first. Then, the simulation model of the flow and heat transfer in the vertical cooler was established by taking the particle movement into account based on the porous medium model and local non-thermodynamic equilibrium double energy equations, and the operational parametric effects, involving the sinter inlet temperature (SIT), air flow rate (AFR) and air inlet temperature (AIT), on the energy and exergy transfer processes in the vertical cooler were investigated in detail. Finally, the net exergy efficiency in the vertical cooler was selected as the indicator function, and the suitable operational parameters of the vertical cooler were determined through the calculation method of the whole working condition. The research results mentioned above 
are very important and useful for improving the recovery rate of sinter waste heat and could provide theoretical guidance for the design and operation of sinter vertical coolers.

\section{Analysis of Energy and Exergy Efficiency}

The analysis methods of energy and exergy efficiency are based on the first and second laws of thermodynamics, which can better analyze and explain the work capacity of a working medium in the case of deviation from the environmental state; obtain the amount of energy loss and reveal the essence of energy loss inside the system; and provide guiding significance for the thermodynamic optimization of whole systems [23].

According to the first law of thermodynamics, the quantity of SWHR is described below.

$$
Q_{w}=m_{\mathrm{g}} c_{\mathrm{g}}\left(T_{\mathrm{g}, \mathrm{out}}-T_{\mathrm{g}, \text { in }}\right)
$$

The energy efficiency is an important index to evaluate the energy recovery effect of a system, which refers to the proportion of the quantity of SWHR that is further utilized in the total input energy. The energy efficiency of the system is expressed as follows.

$$
\eta_{\mathrm{e}}=\frac{Q_{w}}{\sum Q_{\mathrm{in}}} \times 100 \%=\frac{Q_{w}}{Q_{\mathrm{s}, \text { in }}+Q_{\mathrm{g}, \mathrm{in}}} \times 100 \%
$$

where $Q_{\mathrm{s}, \text { in }}$ is the sinter inlet energy, and $Q_{\mathrm{g} \text {,in }}$ is the gas inlet energy, which are given below.

$$
\begin{aligned}
& Q_{\mathrm{s}, \text { in }}=m_{\mathrm{s}} c_{\mathrm{s}}\left(T_{\mathrm{s}, \text { in }}-T_{0}\right) \\
& Q_{\mathrm{g}, \text { in }}=m_{\mathrm{g}} c_{\mathrm{g}}\left(T_{\mathrm{g}, \text { in }}-T_{0}\right)
\end{aligned}
$$

When the sinter temperature is constant, the expression of the sinter exergy value is described below based on the second law of thermodynamics.

$$
E x_{\mathrm{s}}=m_{\mathrm{s}}\left(\int_{T_{0}}^{T_{\mathrm{s}}} c_{\mathrm{s}} \mathrm{d} T-T_{0} \int_{T_{0}}^{T_{\mathrm{s}}} \frac{c_{\mathrm{s}}}{T} \mathrm{~d} T\right)=m_{\mathrm{s}} \mathcal{c}_{\mathrm{s}}\left(T_{\mathrm{s}}-T_{0}\right)\left[1-\frac{T_{0}}{T_{\mathrm{s}}-T_{0}} \ln \frac{T_{\mathrm{s}}}{T_{0}}\right]
$$

When the gas temperature is constant, the expression of the gas exergy value is described below.

$$
E x_{\mathrm{g}}=m_{\mathrm{g}}\left(\int_{T_{0}}^{T_{\mathrm{g}}} c_{\mathrm{g}} \mathrm{d} T-T_{0} \int_{T_{0}}^{T_{\mathrm{g}}} \frac{c_{\mathrm{g}}}{T} \mathrm{~d} T\right)=m_{\mathrm{g}} c_{\mathrm{g}}\left(T_{\mathrm{g}}-T_{0}\right)\left[1-\frac{T_{0}}{T_{\mathrm{g}}-T_{0}} \ln \frac{T_{\mathrm{g}}}{T_{0}}\right]
$$

When the cooling air passes through the vertical cooler, an air pressure drop is bound to occur due to the influence of the sinter particles on the gas flow in the bed layer, which involves viscous resistance loss and inertia resistance loss. The energy loss of the gas flow in a vertical cooler can be described quantitatively by the air pressure exergy because the air outlet pressure in a vertical cooler is the ambient pressure. The air pressure exergy is given below [12].

$$
E x_{\mathrm{g}, \mathrm{p}}=m_{\mathrm{g}} R_{\mathrm{g}} T_{0} \int_{p_{0}}^{p_{\text {in }}} \frac{\mathrm{d} p}{p}=m_{\mathrm{g}} R_{\mathrm{g}} T_{0} \ln \frac{p_{\mathrm{in}}}{p_{0}}=m_{\mathrm{g}} R_{\mathrm{g}} T_{0} \ln \frac{p_{0}+\Delta p}{p_{0}}
$$

As it is known from Equation (3), because the air pressure drop in the bed layer is larger than zero, the air pressure exergy is also greater than zero, which means that the gas flow in the vertical cooler needs to consume the external work. Therefore, the air net exergy in a vertical cooler is defined as the difference between the air outlet exergy and air pressure exergy, which can be determined below.

$$
\begin{gathered}
E x_{\mathrm{g}, \text { net }}=E x_{\mathrm{g}, \text { out }}-E x_{\mathrm{g}, \mathrm{p}} \\
E x_{\mathrm{g}, \text { net }}=m_{\mathrm{g}} c_{\mathrm{g}}\left(T_{\mathrm{g}, \text { out }}-T_{0}\right)\left[1-\frac{T_{0}}{T_{\mathrm{g}, \text { out }}-T_{0}} \ln \frac{T_{\mathrm{g}, \text { out }}}{T_{0}}\right]-m_{\mathrm{g}} R_{\mathrm{g}} T_{0} \ln \frac{p_{0}+\Delta p}{p_{0}}
\end{gathered}
$$


The net exergy efficiency is an important index to evaluate the energy recovery and utilization effect of a system, which refers to the proportion of the net output exergy that is further utilized in the total input exergy. The higher the net exergy efficiency, the higher the energy recovery and utilization efficiency of the system. The net exergy efficiency is expressed as follows.

$$
\eta_{\mathrm{ex}}=\frac{E x_{\mathrm{g}, \mathrm{net}}}{\sum E x_{\mathrm{in}}} \times 100 \%=\frac{E x_{\mathrm{g}, \mathrm{out}}-E x_{\mathrm{g}, \mathrm{p}}}{E x_{\mathrm{s}, \mathrm{in}}+E x_{\mathrm{g}, \text { in }}} \times 100 \%
$$

\section{Description of Simulation Model}

\subsection{Physical Model}

The structure diagram of a vertical cooler for SWHR is revealed in Figure 1. The whole body of the vertical cooler is composed of five parts: feeding section, pre-storage section, ramps section, cooling section and discharging section. Based on previous research results from a vertical cooler [24], the heat exchange of the cooling air with the sinter particles in the bed layer mainly occurs in the cooling section, meaning the cooling section of the vertical cooler was set as the physical model and simulation area in the modeling process.

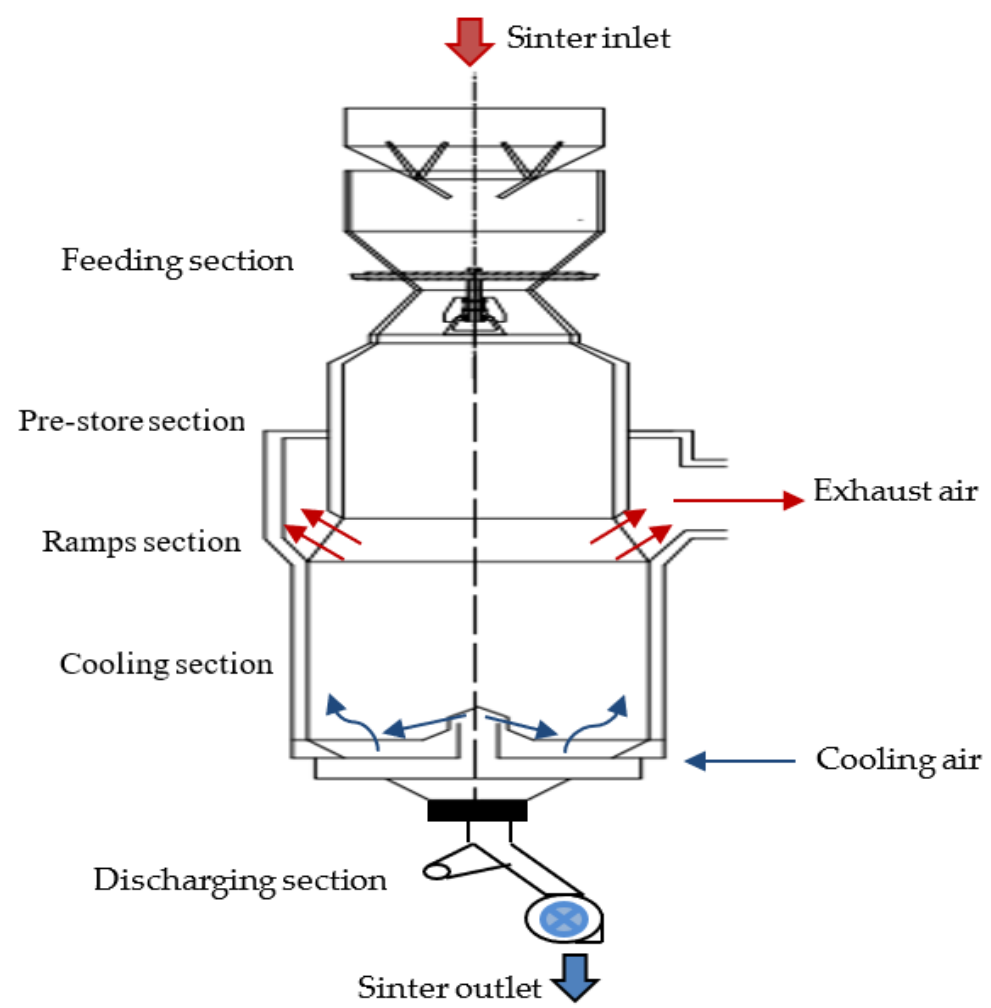

Figure 1. Schematic diagram of vertical cooler for SWHR.

As the sinter particles are different in size and irregular in shape, on the premise of ensuring the accuracy of numerical calculation, the sinter bed layer in the vertical cooler can be simplified. At present, the commonly used method is to conduct average and statistical treatments for the flow and heat transfer in the vertical cooler and regard the cooling section of the vertical cooler as the porous medium region [25]. Therefore, the physical model of the vertical cooler can be simplified as follows [7,9].

(1) The vertical cooler runs under stable working conditions, the operating parameters of which are constant;

(2) The sinter bed layer in the vertical cooler is a homogeneous and isotropic porous medium, without considering the sinter porosity and deformation at high temperature; 
(3) The radiation heat transfer between the particles and the gas in the bed layer is neglected, as well as the heat dissipation of the vertical cooler.

\subsection{Mathematical Description}

According to the Navier-Stokes equation of incompressible viscous fluids, the standard $k-\varepsilon$ turbulence model is applied to analyze the gas flow law in vertical coolers [26]. The calculation of the heat transfer in vertical coolers is performed through local nonthermodynamic equilibrium double energy equations [27], and the governing equations of mass conservation, momentum conservation and energy conservation in vertical coolers are as follows $[28,29]$.

(1) Mass conservation equation

$$
\nabla \cdot\left(\rho_{\mathrm{g}} \mathbf{u}_{\mathrm{g}}\right)=0
$$

(2) Momentum conservation equation

$$
\rho_{\mathrm{g}}\left(\mathbf{u}_{\mathrm{g}} \cdot \nabla\right) \mathbf{u}_{\mathrm{g}}=\nabla \cdot\left[-p \mathbf{I}+\left(\mu+\mu_{\mathrm{T}}\right)\left(\nabla \mathbf{u}_{\mathrm{g}}+\left(\nabla \mathbf{u}_{\mathrm{g}}\right)^{\mathrm{T}}\right)-\frac{2}{3}\left(\mu+\mu_{\mathrm{T}}\right)\left(\nabla \cdot \mathbf{u}_{\mathrm{g}}\right) \mathbf{I}-\frac{2}{3} \rho_{\mathrm{g}} k \mathbf{I}\right]+\mathbf{F}+\mathbf{S}
$$

In order to analyze the gas flow in a porous medium, a source term $\mathbf{S}$ is added to the right side of the equation to modify the momentum transfer equation in a porous medium, and the expression of the source term is shown as follows [7].

$$
\mathbf{S}=-\left(\frac{\mu}{\alpha} \mathbf{u}_{\mathrm{g}}+\frac{1}{2} C_{2} \rho_{\mathrm{g}}\left|u_{\mathrm{g}}\right| \mathbf{u}_{\mathrm{g}}\right)
$$

where $1 / a$ is the viscous resistance coefficient, and $C_{2}$ is the inertial resistance coefficient, which are given below, derived from previous research results [30].

$$
\begin{aligned}
& \frac{1}{a}=\left[85.4+3294 e^{\left(-0.085 D / d_{\mathrm{p}}\right)}\right] \frac{(1-\varepsilon)^{2}}{\varepsilon^{3} d_{\mathrm{p}}{ }^{2}} \\
& C_{2}=\left[0.632+2.8 e^{\left(-0.112 D / d_{\mathrm{p}}\right)}\right] \frac{(1-\varepsilon)}{\varepsilon^{3} d_{\mathrm{p}}}
\end{aligned}
$$

(3) Energy conservation equations

Due to the great difference in the thermal conductivity and heat capacity of the two gas-solid phases in the sinter bed layer, the gas-solid temperature difference at the same height in the bed layer is obvious [9]. Therefore, local non-thermodynamic equilibrium double energy equations are used to solve the gas-solid heat transfer in the cooling section of the vertical cooler. The specific expressions of the energy equations are given below [29].

Sinter:

$$
\rho_{\mathrm{s}} \mathcal{c}_{\mathrm{s}} u_{\mathrm{s}} \cdot \nabla T_{\mathrm{s}}=(1-\varepsilon) \nabla \cdot\left(\lambda_{\mathrm{s}} \nabla T_{\mathrm{s}}\right)-h_{\mathrm{v}}\left(T_{\mathrm{s}}-T_{\mathrm{g}}\right)
$$

Cooling air:

$$
\rho_{\mathrm{g}} c_{\mathrm{g}} u_{\mathrm{g}} \cdot \nabla T_{\mathrm{g}}=\varepsilon \nabla \cdot\left(\lambda_{\mathrm{g}} \nabla T_{\mathrm{g}}\right)+h_{\mathrm{v}}\left(T_{\mathrm{s}}-T_{\mathrm{g}}\right)
$$

where $h_{\mathrm{v}}$ is the volume heat transfer coefficient in the sinter bed layer, which can be determined by the following expression [31].

$$
h_{\mathrm{v}}=\frac{6 h(1-\varepsilon)}{d_{\mathrm{p}}}=\frac{N u \lambda_{\mathrm{g}}}{d_{\mathrm{p}}} \frac{6(1-\varepsilon)}{d_{\mathrm{p}}}
$$

where $h$ is the convective heat transfer coefficient, and $N u$ is the gas-solid heat transfer Nusselt number, which is determined below, derived from previous research results [32].

$$
N u=0.198 \varepsilon^{0.07} \operatorname{Re}^{0.66} \operatorname{Pr}^{1 / 3}
$$


where $R e$ is the Reynolds number, and $P r$ is the Prandtl number, the expressions of which are given below.

$$
\begin{gathered}
R e=\frac{\rho_{\mathrm{g}} u_{\mathrm{g}} d_{\mathrm{p}}}{\mu} \\
\operatorname{Pr}=\frac{\mathrm{c}_{\mathrm{g}} \mu}{\lambda_{\mathrm{g}}}
\end{gathered}
$$

\subsection{Grid Generation and Boundary Conditions}

In the present work, the ICEM-CFD software was used to perform the geometry construction and mesh generation of the simulation area mentioned in Figure 1 . As the research object, namely, the cooling section of a vertical tank, is a symmetric cylinder, the structured grid fits the grid generation with a uniform symmetrical structure perfectly. The structured grid only contains quadrilateral elements and a hexahedron. Compared with the unstructured grid, the structured grid has many advantages, such as the realization of easy boundary fitting, a faster grid generation speed, a better quality of grid generation and the smaller memory of the computing run. Therefore, a three-dimensional structured grid with quadrilateral elements was determined for the mesh generation of the simulation area $[33,34]$, and the generation result of the simulation area is shown in Figure 2.

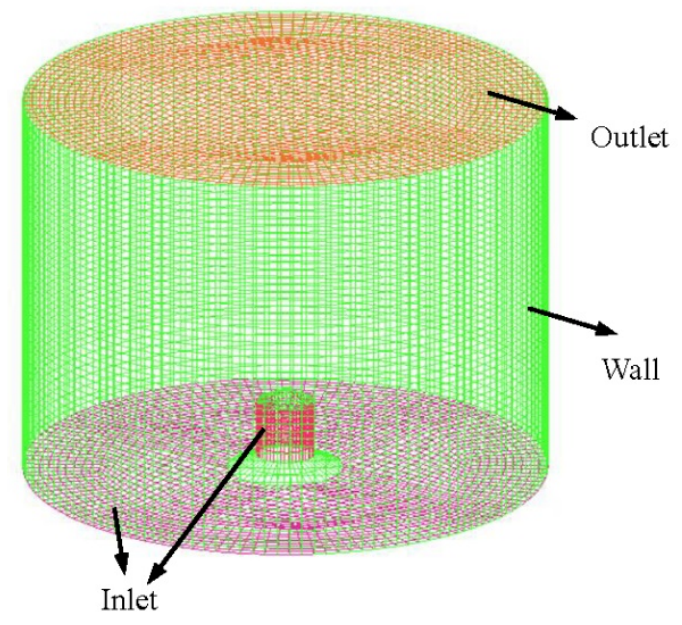

Figure 2. Mesh generation of simulation area.

The FLUENT-CFD software was applied to calculate the flow and heat transfer in the simulation area. The user-defined functions (UDFs) in the FLUENT-CFD software were developed to define and compile the gas-solid physical parameters, source term $S_{i}$ and heat transfer coefficient $h$. Furthermore, the convective term with the particle movement was also compiled through the transition of the source term in the solid energy equation.

The boundary conditions for the simulation area were set as shown in Figure 2. As a condition of the gas inlet boundary, the mass flow condition was adopted for the gas inlet of the bottom and central cowl in the simulation area. Furthermore, the pressure outlet condition with a gas relative pressure of zero was used for the gas outlet of the top in the simulation area. In addition, the wall of the simulation area was set as the adiabatic condition, which means that the heat loss in the wall was not considered.

\subsection{Model Verification}

As vertical coolers are still in the stages of theoretical and experimental studies, a selfmade experimental cooler for the measurement of the experimental data was developed, and the structural diagram of the experimental cooler is shown in Figure 3. The reliability verification of the above simulation model was performed by contrastive analysis between the simulation values and the measurement data. The related parameters of the sinter and the environment used in the simulation $[35,36]$ are listed in Table 1 . The air outlet 
temperature was selected as the verification function of the simulation model, and the contrastive results of five operational cases are presented in Table 2.

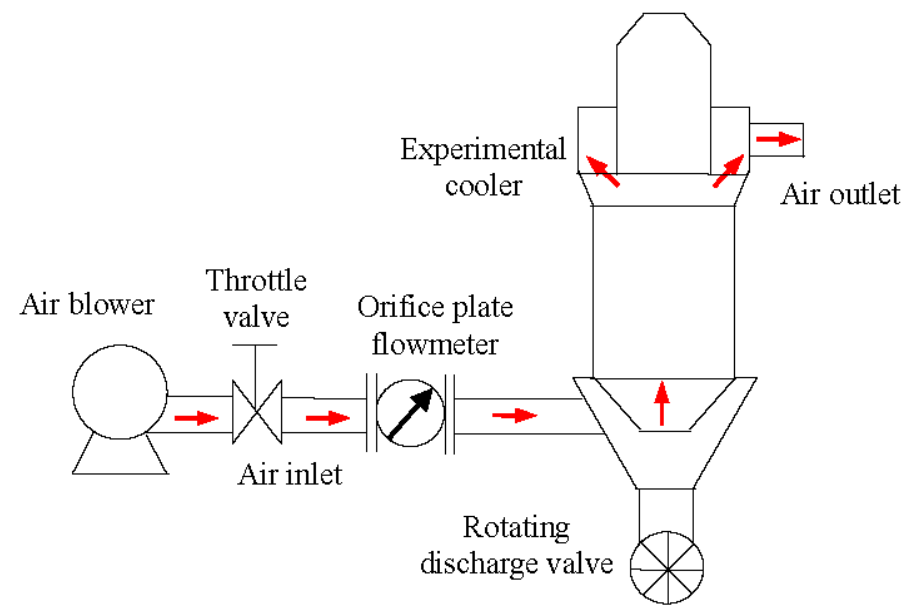

Figure 3. Structural diagram of the experimental cooler.

Table 1. Related parameters of the sinter and the environment.

\begin{tabular}{cc}
\hline Parameters & Values \\
\hline$d_{\mathrm{p}}(\mathrm{m})$ & 0.035 \\
$c_{\mathrm{s}}(\mathrm{J} /(\mathrm{kg} \cdot \mathrm{K}))$ & $337.03\left(T_{\mathrm{s}}-273\right)^{0.152}$ \\
$\lambda_{\mathrm{s}}(\mathrm{w} /(\mathrm{m} \cdot \mathrm{K}))$ & 2.87 \\
$\rho_{\mathrm{s}}\left(\mathrm{kg} / \mathrm{m}^{3}\right)$ & 3400 \\
$\varepsilon$ & 0.41 \\
$T_{0}(\mathrm{~K})$ & 293 \\
$p_{0}(\mathrm{~Pa})$ & 101,325 \\
\hline
\end{tabular}

Table 2. Contrastive results of five operational cases.

\begin{tabular}{|c|c|c|c|c|c|c|c|}
\hline \multirow[b]{2}{*}{ Cases } & \multirow[b]{2}{*}{$\operatorname{SIT}\left({ }^{\circ} \mathrm{C}\right)$} & \multirow[b]{2}{*}{$\operatorname{AFR}\left(\mathrm{m}^{3} / \mathrm{h}\right)$} & \multirow{2}{*}{$\begin{array}{l}\text { Sinter Flow } \\
\text { Rate }(\mathrm{kg} / \mathrm{h})\end{array}$} & \multirow[b]{2}{*}{ AIT $\left({ }^{\circ} \mathrm{C}\right)$} & \multicolumn{3}{|c|}{ Air Outlet Temperature } \\
\hline & & & & & $\begin{array}{l}\text { Measurement } \\
\text { Value }\left({ }^{\circ} \mathrm{C}\right)\end{array}$ & $\begin{array}{l}\text { Simulation } \\
\text { Value }\left({ }^{\circ} \mathrm{C}\right)\end{array}$ & $\begin{array}{c}\text { Deviation } \\
(\%)\end{array}$ \\
\hline 1 & 769.2 & 2127 & 2520 & 20 & 504.5 & 481.6 & -4.56 \\
\hline 2 & 873.6 & 1723 & 2160 & 20 & 631.3 & 594.5 & -5.83 \\
\hline 3 & 720.9 & 1684 & 2160 & 20 & 502.4 & 478.6 & -4.74 \\
\hline 4 & 732.7 & 1545 & 1800 & 20 & 477.9 & 454.2 & -4.96 \\
\hline 5 & 781.4 & 1499 & 1800 & 20 & 543.1 & 518.3 & -4.57 \\
\hline
\end{tabular}

As it can be seen in Table 2, the simulation values of the air outlet temperature under the five cases match well with the measurement values, and the average calculation deviation between the simulation values and the measurement values is $4.93 \%$, which means that the above simulation model and analytical methods for the cooling section of the vertical cooler are credible and correct.

\section{Results and Discussion}

The actual sinter production of a $360 \mathrm{~m}^{2}$ sintering machine was taken as the simulation processing capacity of the vertical cooler, namely, 3.9 million tons per year, which means that the sinter mass flow rate in the vertical cooler is $152 \mathrm{~kg} / \mathrm{s}$. The inner diameter and height of the cooling section were set as $9 \mathrm{~m}$ and $7 \mathrm{~m} \mathrm{[37],} \mathrm{respectively,} \mathrm{and} \mathrm{the} \mathrm{related}$ parameters of the sinter and the environment used in the simulation are also listed in Table 1. Due to the difference in the sintering terminal point position on the sintering 
machine and the preheating effect of the gas inlet at the bottom of the vertical cooler, the SIT and AIT entering into the vertical cooler are not constant, and the AFR is also an important factor affecting the flow and heat transfer in the vertical cooler. Therefore, based on the established simulation model mentioned above, and according to the three main factors affecting the flow and heat transfer in the vertical cooler, namely, the SIT, AFR and AIT, the influence law of the third factor on the energy and exergy transfer processes in the vertical cooler was calculated and analyzed in detail when the set values of the two other factors were invariable. On this basis, the net exergy efficiency of the vertical cooler was identified as the indicator function of the parameter optimization, and the parameter optimization analysis of the vertical cooler was carried out. The change conditions of the three influencing factors are shown in Table 3.

Table 3. Change conditions of three influencing factors.

\begin{tabular}{cccc}
\hline Levels & $\boldsymbol{T}_{\mathbf{s , \text { in }}}(\mathbf{K})$ & $\boldsymbol{m}_{\mathbf{g}} \mathbf{( \mathbf { k g } / \mathbf { s } )}$ & $\boldsymbol{T}_{\mathbf{g}, \text { in }}(\mathbf{K})$ \\
\hline 1 & 873 & 170 & 293 \\
2 & 898 & 180 & 313 \\
3 & 923 & 190 & 333 \\
4 & 948 & 200 & 353 \\
5 & 973 & 210 & 373 \\
\hline
\end{tabular}

\subsection{Effect of SIT}

By setting the AFR and AIT to $190 \mathrm{~kg} / \mathrm{s}$ and $293 \mathrm{~K}$, respectively, the variations in the air outlet temperature, sinter outlet temperature and air pressure drop in the sinter bed layer were determined for various SITs, as shown in Figure 4.

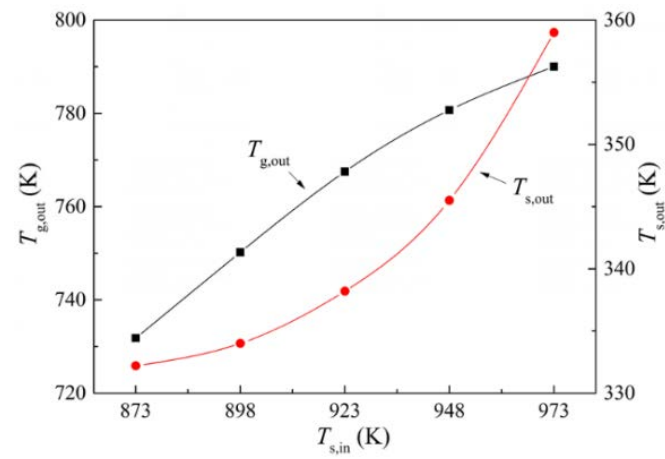

(a) $T_{\mathrm{g}, \text { out }}$ and $T_{\mathrm{s} \text {,out }}$

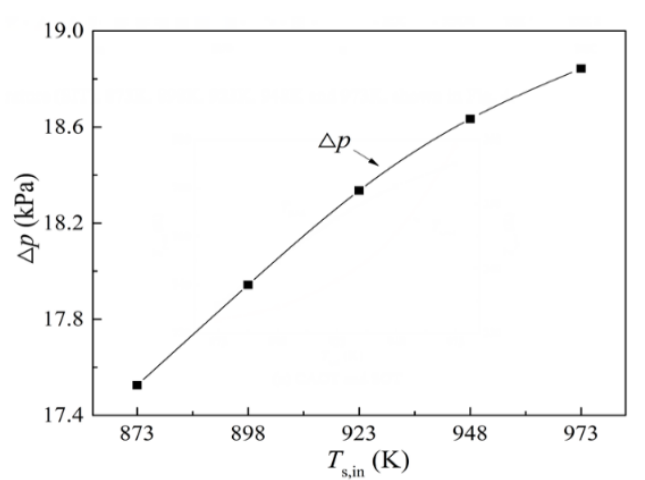

(b) $\Delta p$

Figure 4. Effect of SIT on air outlet temperature, sinter outlet temperature and air pressure drop.

As it can be seen in Figure 4, with the increase in the SIT, the air outlet temperature, sinter outlet temperature and air pressure drop gradually increase. This may be explained as follows: the heat transfer temperature difference in the sinter with the air in the bed layer gradually increases as the SIT increases, and then the heat transfer quantity also increases, which leads to the increase in the air outlet temperature for the given AFR. As the sinter output and AFR are constant, the heat exchange time of the sinter with the air in the bed layer is also constant, and due to the increase in the SIT, the sinter waste heat carried into the vertical tank is not taken away completely by the air, meaning the sinter outlet temperature also increases as the SIT increases. Furthermore, the enhancement of the gas-solid heat transfer results in an increase in the air temperature in the bed layer, the gas velocity in the bed layer also increases accordingly, which results in the intensified collision between the cooling air and sinter particles, and the inertia force of the gas flow in the bed layer also increases, meaning the air pressure drop gradually increases.

The variation in the quantity of SWHR and energy efficiency for various SITs was obtained and is revealed in Figure 5. As it can be seen in Figure 5, the quantity of SWHR 
increases as the SIT increases, while the energy efficiency gradually decreases. This may be explained as follows: with the increase in the SIT, the increase in the air outlet temperature results in an increase in the quantity of SWHR. The increased amplitude of the sinter outlet temperature shown in Figure 4 becomes larger and larger with the increase in the SIT, which results in a decrease in energy efficiency.

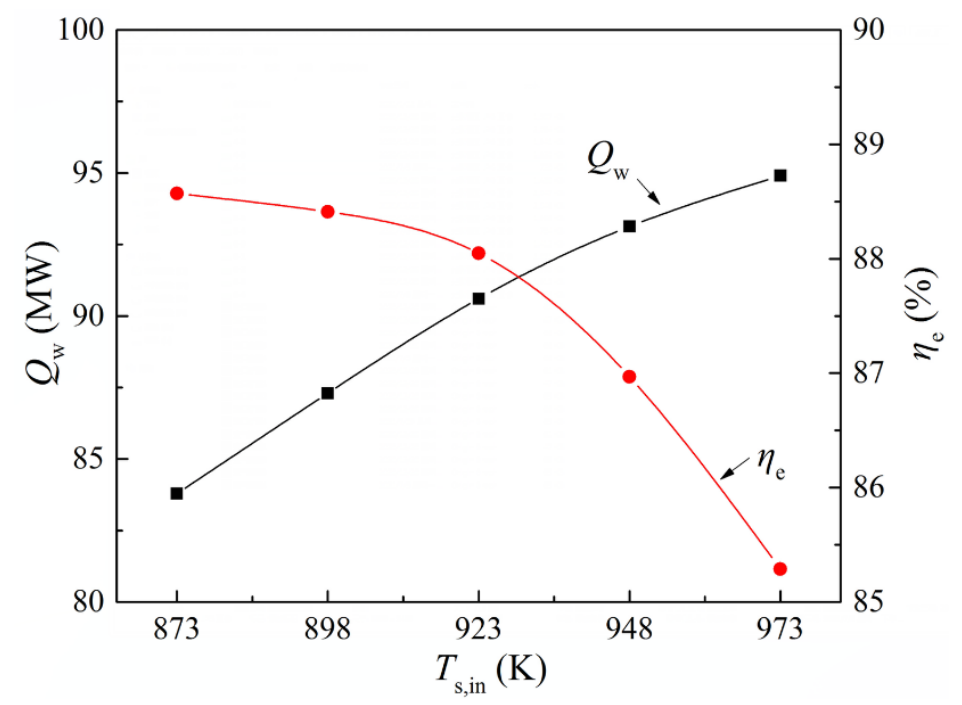

Figure 5. Effect of SIT on quantity of SWHR and energy efficiency.

Figure 6 reveals the changes in the air outlet exergy and air pressure exergy in the sinter bed layer with the SIT. As it can be seen in Figure 6, both the air outlet exergy and air pressure exergy increase as the SIT increases. That is to say, the increase in the SIT results in the increase in the air outlet exergy and air pressure exergy for a given AFR, and according to Equations (6) and (7), the air outlet exergy and air pressure exergy also gradually increase.

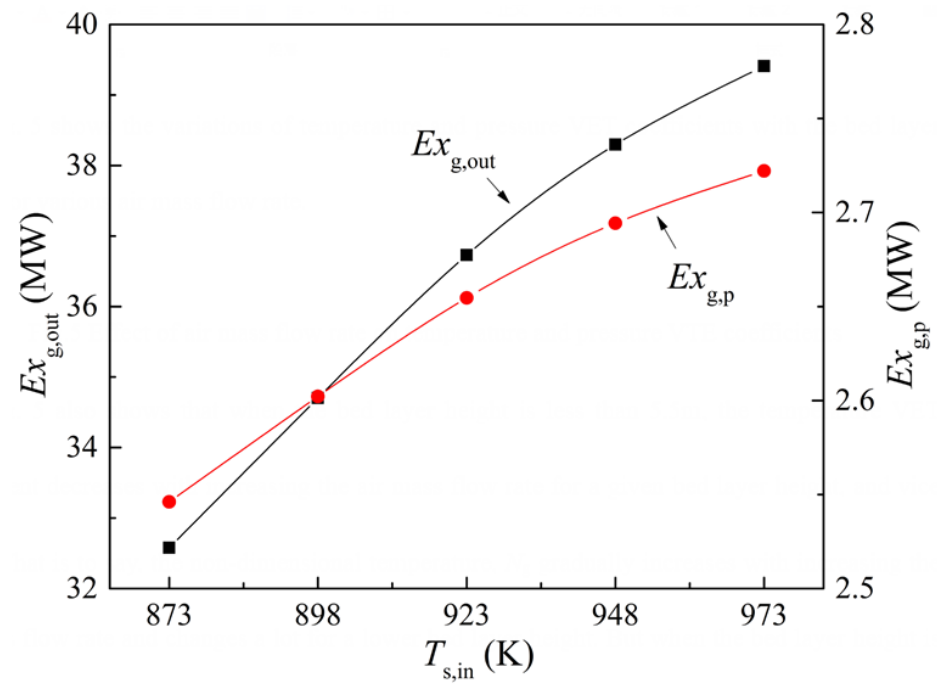

Figure 6. Effect of SIT on air outlet exergy and air pressure exergy.

The variation in the air net exergy and net exergy efficiency for various SITs was obtained and is revealed in Figure 7. As it can be seen in Figure 7, with the increase in the SIT, the air net exergy gradually increases, and the net exergy efficiency gradually decreases. That is to say, because the value of the air outlet exergy is always greater than that of the air pressure exergy with the increase in the SIT, the air net exergy also increases based on Equation (8). Furthermore, the increase in the SIT leads to the increase in the 
sinter outlet temperature, the sinter outlet exergy also increases based on Equation (5) and the exergy loss in the vertical cooler gradually increases, which leads to the decrease in the net exergy efficiency.

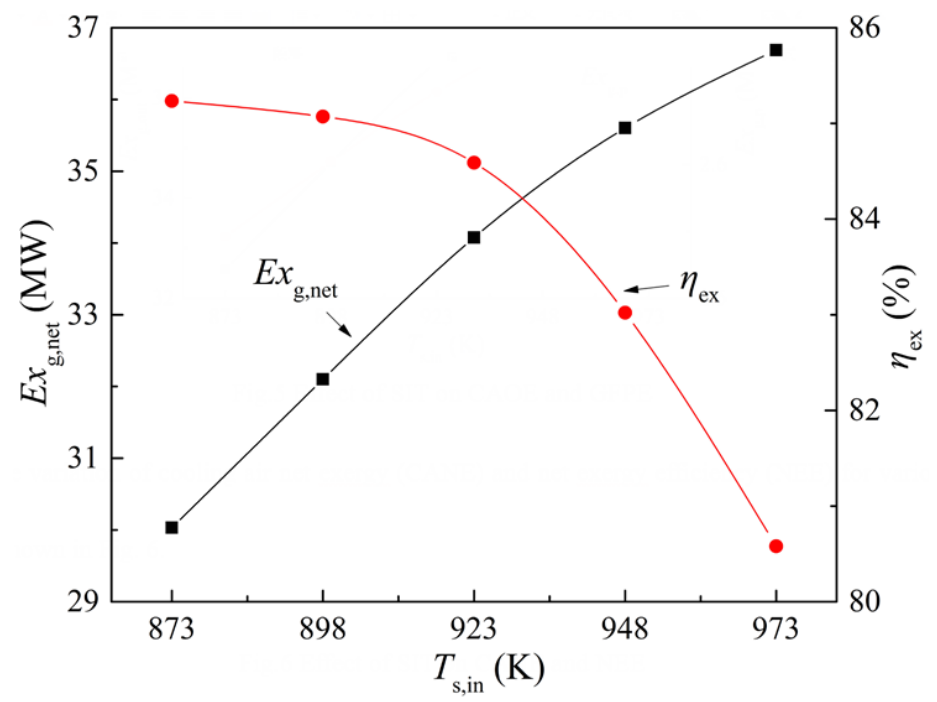

Figure 7. Effect of SIT on air net exergy and net exergy efficiency.

\subsection{Effect of AFR}

By setting the AIT and SIT to $293 \mathrm{~K}$ and $923 \mathrm{~K}$, respectively, the variations in the air outlet temperature, sinter outlet temperature and air pressure drop in the bed layer were obtained and are shown in Figure 8 for various AFRs. As it can be seen in Figure 8, both the air outlet temperature and sinter outlet temperature gradually decrease, and the air pressure drop gradually increases as the AFR increases. This may be explained as follows: when the sinter inlet heat flux is constant, the air outlet temperature decreases with the increasing AFR based on the energy conservation law. Furthermore, the heat transfer quantity of the sinter with the cooling air in the bed layer increases as the AFR increases, and the sinter cooling rate also increases accordingly, which results in the decrease in the sinter outlet temperature. Moreover, the increase in the AFR leads to the increase in the gas velocity, and the viscosity and inertia resistance loss in the bed layer also increase, meaning the air pressure drop increases accordingly.

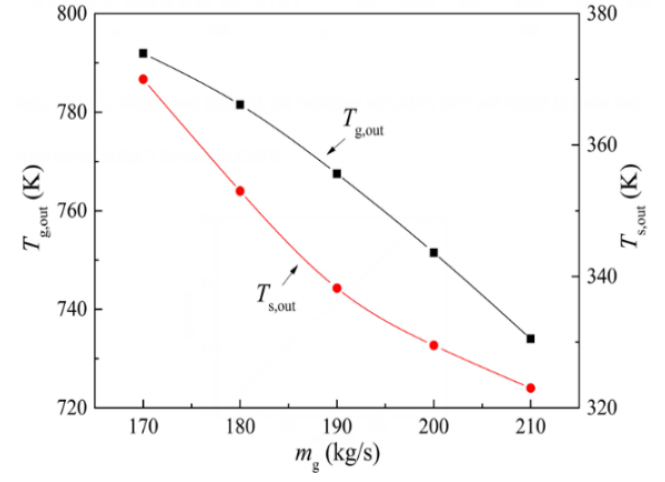

(a) $T_{\text {g,out }}$ and $T_{\mathrm{s} \text {,out }}$

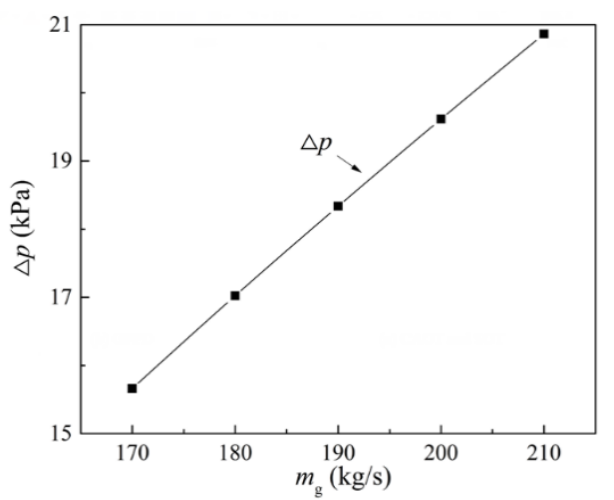

(b) $\Delta p$

Figure 8. Effect of AFR on air outlet temperature, sinter outlet temperature and air pressure drop.

Figure 9 shows the variations in the quantity of SWHR and energy efficiency in the bed layer with the AFR. As it can be seen in Figure 9, both the quantity of SWHR and energy efficiency increase as the AFR increases. That is to say, because the sinter mass flow 
rate and inlet temperature are constant, the decrease in the sinter outlet temperature shown in Figure 8 inevitably results in an increase in the quantity of SWHR and energy efficiency.

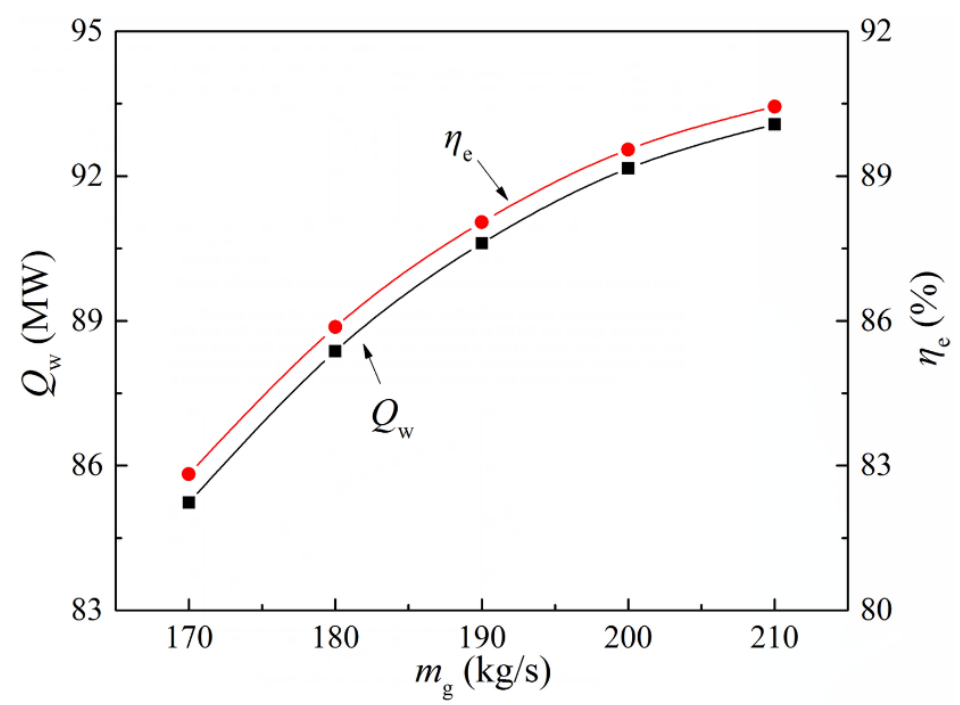

Figure 9. Effect of AFR on quantity of SWHR and energy efficiency.

Figure 10 shows the variations in the air outlet exergy and air pressure exergy in the bed layer with the AFR.

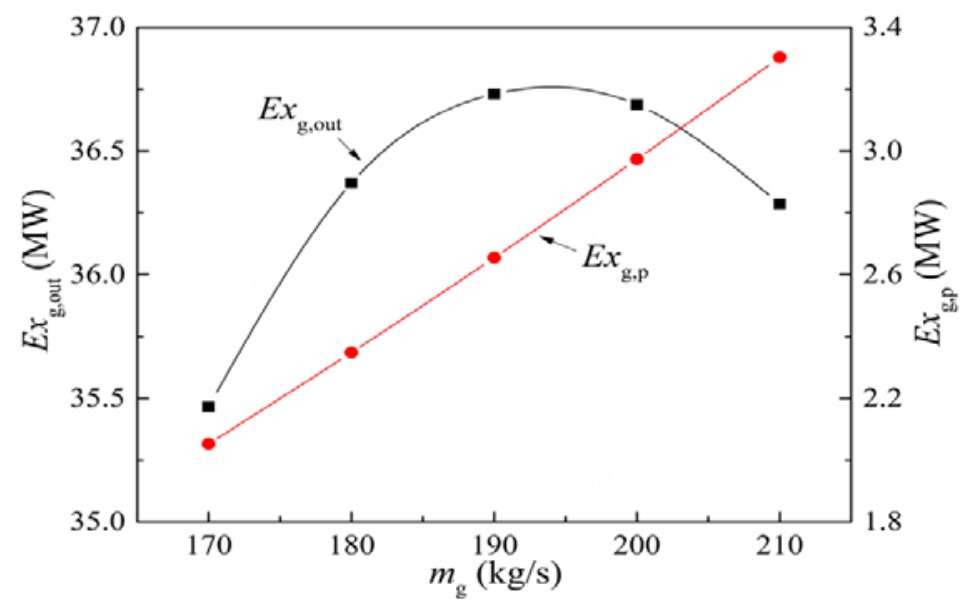

Figure 10. Effect of AFR on air outlet exergy and air pressure exergy.

As it can be seen in Figure 10, the air outlet exergy first increases and then decreases as the AFR increases, while the air pressure exergy increases accordingly. That is to say, when the sinter inlet heat flux is constant, the air outlet heat quantity increases as the sinter outlet temperature decreases, and due to the limitation of the sinter outlet temperature, the increased amplitude of the air outlet heat quantity gradually decreases. As the air outlet temperature decreases as the AFR increases, the air energy level also decreases accordingly. Furthermore, the lower the air outlet temperature, the larger the decrease in the amplitude of the air energy level. As the air outlet exergy is the product of the air outlet heat quantity and the air energy level, the change curve of the air outlet exergy could have a maximum value as the AFR increases. Moreover, the increase in the AFR leads to an increase in the air pressure drop in the bed layer, and the air pressure exergy also increases accordingly.

The variation in the air net exergy and net exergy efficiency for various AFRs was obtained and is revealed in Figure 11. As it can be seen in Figure 11, both the air net exergy and net exergy efficiency first increase and then decrease as the AFR increases. That is to say, because the value of the air outlet exergy is always greater than that of the air pressure 
exergy with the increase in the AFR, as shown in Figure 10, the variation trend of the air net exergy is the same as that of the air outlet exergy. In addition, there is a small increase in the inlet exergy of the cooling air due to the increase in the AFR, which also leads to a small increase in the inlet exergy of the vertical cooler, namely, the value of the denominator in Equation (10) for a given sinter inlet heat flux, meaning the net exergy efficiency also first increases and then decreases as the AFR increases.

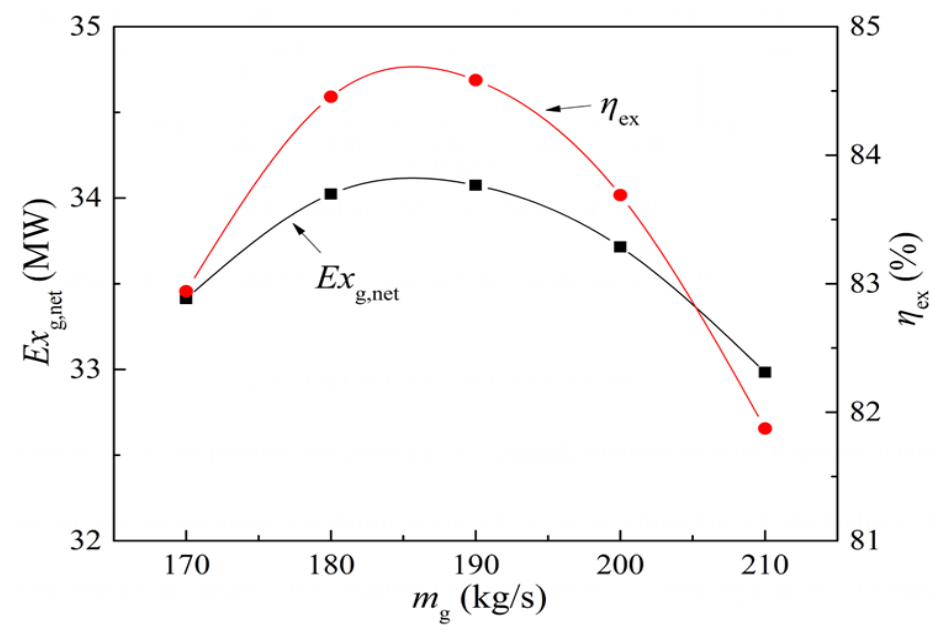

Figure 11. Effect of AFR on air net exergy and net exergy efficiency.

\subsection{Effect of AIT}

By setting the AFR and SIT to $190 \mathrm{~kg} / \mathrm{s}$ and $923 \mathrm{~K}$, respectively, the variations in the air outlet temperature, sinter outlet temperature and air pressure drop in the bed layer were determined for various AITs, which are revealed in Figure 12. As it can be seen in Figure 12, the air outlet temperature, sinter outlet temperature and air pressure drop gradually increase with the increase in the AIT. That is to say, the increase in the AIT leads to the increase in the gas velocity in the bed layer for a given AFR, and the heat exchange intensity is also enhanced accordingly, which leads to an increase in the air outlet temperature. Furthermore, the gas-solid temperature difference in the bed layer gradually decreases as the AIT increases, and the heat exchange quantity of the sinter with the cooling air decreases accordingly in the same periods, which results in an increase in the sinter outlet temperature. In addition, because of the increase in the gas velocity in the bed layer, the air pressure drop also increases accordingly.

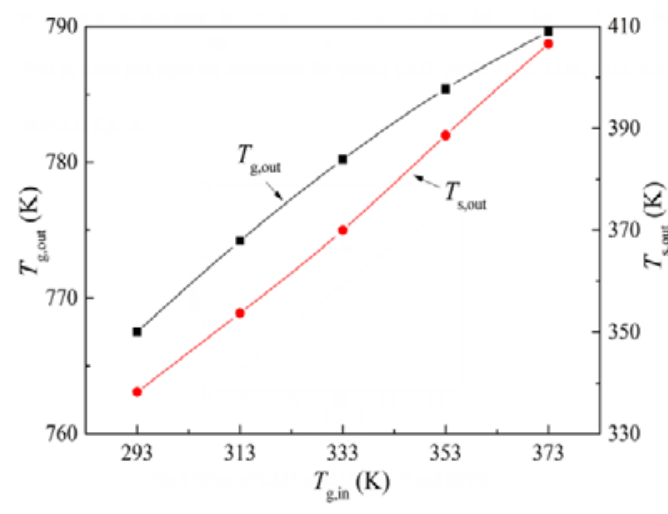

(a) $T_{\mathrm{g} \text {,out }}$ and $T_{\mathrm{s} \text {,out }}$

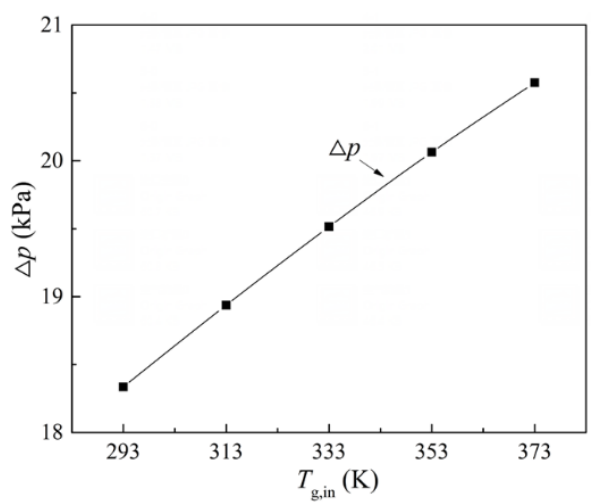

(b) $\Delta p$

Figure 12. Effect of AIT on air outlet temperature, sinter outlet temperature and air pressure drop.

Figure 13 reveals the changes in the quantity of SWHR and energy efficiency in the bed layer with the AIT. As it can be seen in Figure 13, both the quantity of SWHR and energy efficiency gradually decrease with the increase in the AIT. This may be explained as 
follows: since the sinter mass flow rate and inlet temperature are constant, the increase in the sinter outlet temperature, as shown in Figure 12, inevitably results in an increase in the quantity of SWHR and energy efficiency. In addition, the increase in the AIT also results in an increase in the denominator in Equation (7), meaning the decreased amplitude of the energy efficiency is larger than that of the quantity of SWHR.

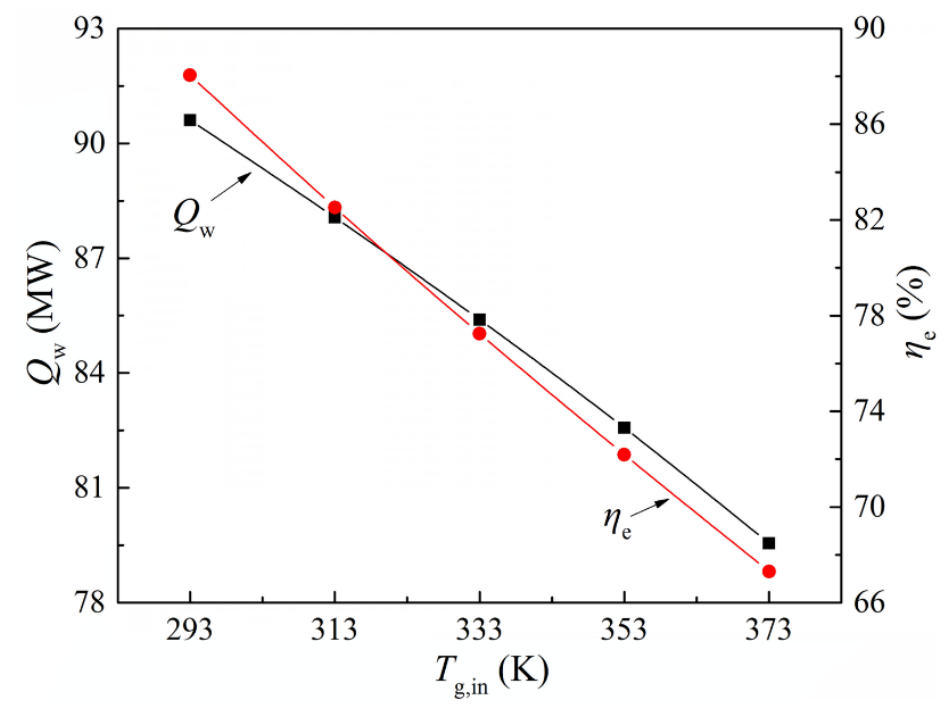

Figure 13. Effect of AIT on quantity of SWHR and energy efficiency.

Figure 14 reveals the changes in the air outlet exergy and air pressure exergy in the sinter bed layer with the AIT. As it can be seen in Figure 14, both the air outlet exergy and air pressure exergy gradually increase with the increase in the AIT. This may be explained as follows: since the increase in the AIT leads to an increase in the air outlet temperature and air pressure drop for a given AFR, according to Equations (6) and (7), the air outlet exergy and air pressure exergy also gradually increase accordingly.

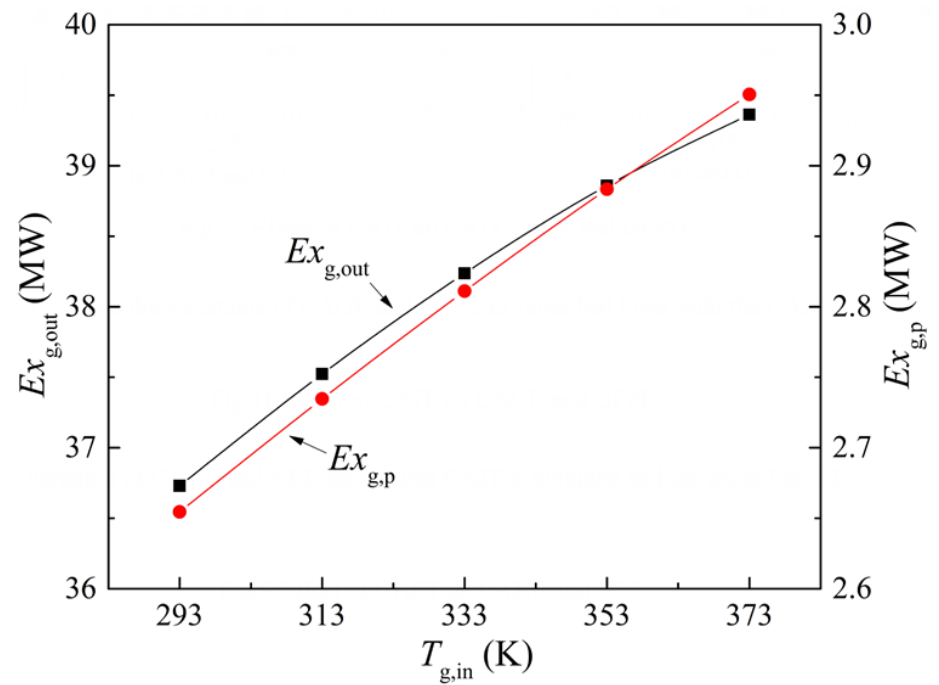

Figure 14. Effect of AIT on air outlet exergy and air pressure exergy.

The variation in the air net exergy and net exergy efficiency for various AITs was obtained and is revealed in Figure 15. As it can be seen in Figure 15, the air net exergy gradually increases with the increase in the AIT, while the net exergy efficiency first increases and then decreases. This may be explained as follows: since the value of the air outlet exergy is always greater than that of the air pressure exergy with the increase in the AIT, as shown in Figure 14, the air net exergy also increases based on Equation (8). 
In addition, the increasing trend of the air outlet exergy shown in Figure 14 is obviously less than that of the air pressure exergy with the increase in the AIT, which results in the increased amplitude of the air net exergy gradually decreasing accordingly. Furthermore, the larger the AIT, the larger the air inlet exergy, which results in a larger increase in the amplitude of the denominator value in Equation (10), meaning the change curve of the net exergy efficiency could have a maximum value as the AIT increases based on Equation (10).

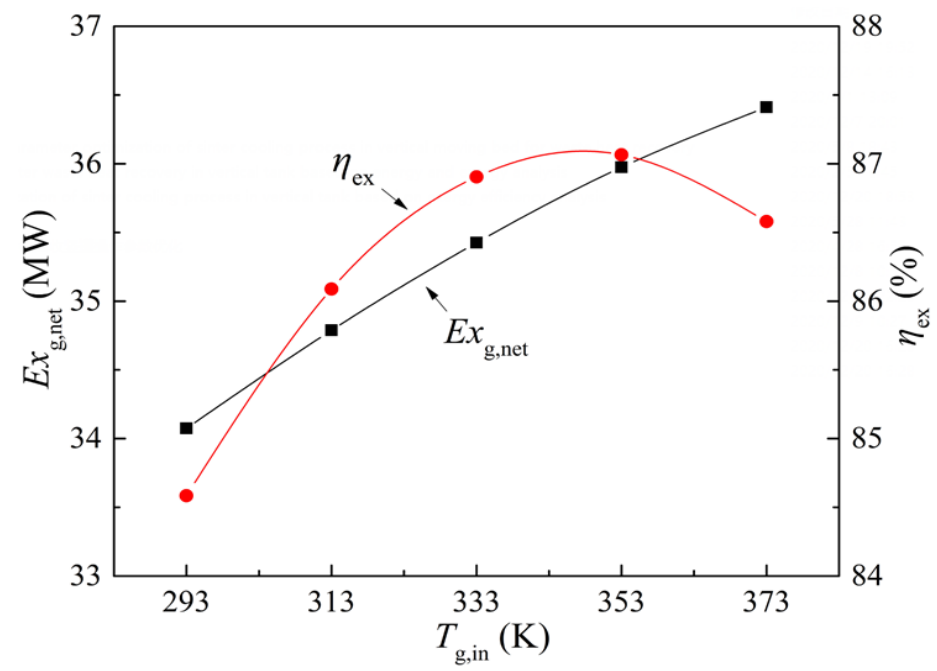

Figure 15. Effect of AIT on air net exergy and net exergy efficiency.

\subsection{Optimization Analysis of Operational Parameter}

According to the analysis of the results of the above three influencing factors, it can be seen that the net exergy efficiency increases monotonically with the increase in the SIT, and with the increasing AFR and AIT, the net exergy efficiency first increases and then decreases. Therefore, only the two parameters of AFR and AIT were selected as the optimized parameters, and the SIT was set to $923 \mathrm{~K}$ for the present optimization simulation. The values of the AFR and AIT at five levels are presented in Table 4.

Table 4. Level table of different operational parameters.

\begin{tabular}{ccc}
\hline Levels & $\boldsymbol{m}_{\mathbf{g}}(\mathbf{k g} / \mathbf{s})$ & $\boldsymbol{T}_{\mathbf{g}, \text { in }}(\mathbf{K})$ \\
\hline 1 & 170 & 293 \\
2 & 180 & 313 \\
3 & 190 & 333 \\
4 & 200 & 353 \\
5 & 210 & 373 \\
\hline
\end{tabular}

In order to obtain the detailed research results, the calculation method of the whole working condition was applied to perform the optimization analysis of two operational parameters, and the net exergy efficiency in the vertical cooler was selected as the optimization objective function. The simulation results of the full working condition are listed in Table 5, and the corresponding calculation values of the net exergy efficiency under different AFRs and AITs are shown in Figure 16. 
Table 5. Simulation results of whole working condition.

\begin{tabular}{|c|c|c|c|c|c|c|}
\hline \multirow{2}{*}{ Conditions } & \multicolumn{2}{|c|}{ Parameter Setting } & \multirow{2}{*}{$T_{\mathrm{g}, \text { out }}(\mathrm{K})$} & \multirow{2}{*}{$\Delta p(\mathbf{k P a})$} & \multirow{2}{*}{$E x_{\mathrm{g}, \text { net }}(\mathrm{MW})$} & \multirow{2}{*}{$\eta_{\mathrm{ex}}(\%)$} \\
\hline & $T_{\mathrm{g}, \text { in }}(\mathrm{K})$ & $m_{\mathrm{g}}(\mathrm{kg} / \mathrm{s})$ & & & & \\
\hline 1 & 293 & 170 & 791.9 & 15.66 & 33.41 & 82.94 \\
\hline 2 & 293 & 180 & 781.5 & 17.02 & 34.02 & 84.46 \\
\hline 3 & 293 & 190 & 767.4 & 18.34 & 34.07 & 84.58 \\
\hline 4 & 293 & 200 & 751.5 & 19.62 & 33.71 & 83.69 \\
\hline 5 & 293 & 210 & 734.1 & 20.86 & 32.98 & 81.87 \\
\hline 6 & 313 & 170 & 798.4 & 16.16 & 34.05 & 84.3 \\
\hline 7 & 313 & 180 & 788.1 & 17.57 & 34.7 & 85.89 \\
\hline 8 & 313 & 190 & 774.2 & 18.94 & 34.79 & 86.08 \\
\hline 9 & 313 & 200 & 758.3 & 20.27 & 34.46 & 85.26 \\
\hline 10 & 313 & 210 & 740.9 & 21.58 & 33.76 & 83.51 \\
\hline 11 & 333 & 170 & 804.2 & 16.63 & 34.62 & 85.05 \\
\hline 12 & 333 & 180 & 794 & 18.09 & 35.31 & 86.67 \\
\hline 13 & 333 & 190 & 780.2 & 19.51 & 35.43 & 86.9 \\
\hline 14 & 333 & 200 & 764.4 & 20.91 & 35.13 & 86.12 \\
\hline 15 & 333 & 210 & 747.1 & 22.27 & 34.45 & 84.41 \\
\hline 16 & 353 & 170 & 809.2 & 17.08 & 35.12 & 85.21 \\
\hline 17 & 353 & 180 & 799.1 & 18.59 & 35.83 & 86.82 \\
\hline 18 & 353 & 190 & 785.4 & 20.06 & 35.97 & 87.06 \\
\hline 19 & 353 & 200 & 769.7 & 21.52 & 35.7 & 86.29 \\
\hline 20 & 353 & 210 & 752.5 & 22.94 & 35.05 & 84.61 \\
\hline 21 & 373 & 170 & 813.4 & 17.49 & 35.52 & 84.85 \\
\hline 22 & 373 & 180 & 803.4 & 19.05 & 36.26 & 86.42 \\
\hline 23 & 373 & 190 & 789.8 & 20.58 & 36.43 & 86.63 \\
\hline 24 & 373 & 200 & 774.2 & 22.09 & 36.18 & 85.85 \\
\hline 25 & 373 & 210 & 757.1 & 23.57 & 35.56 & 84.17 \\
\hline
\end{tabular}

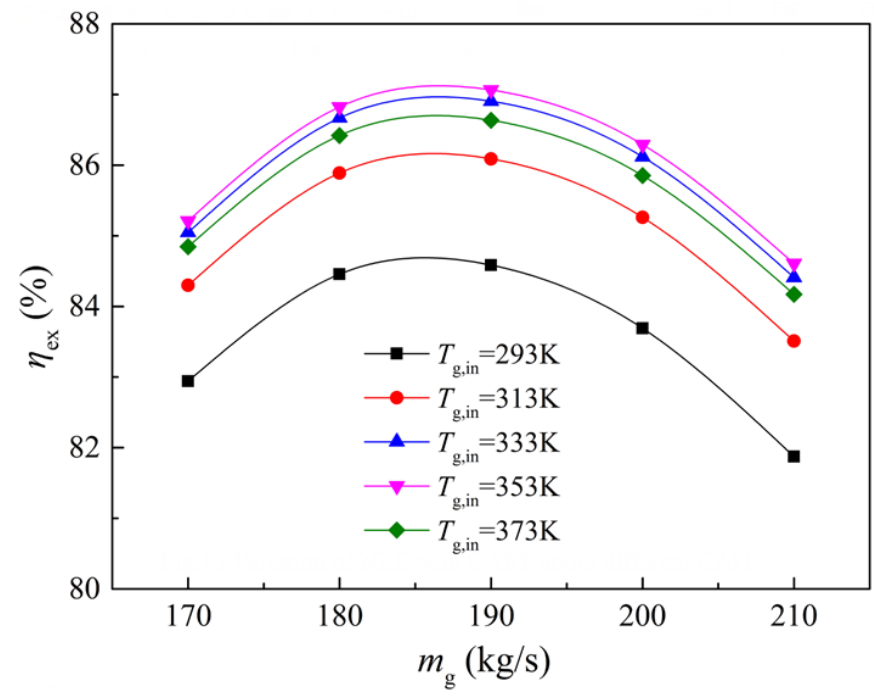

Figure 16. Variation in net exergy efficiency with AFR under different AITs.

As it can be seen from Figure 16, the net exergy efficiency first increases and then decreases as the AFR increases under different AITs, while the net exergy efficiency also first increases and then decreases as the AIT increases for a given AFR. When the AIT is $353 \mathrm{~K}$, the net exergy efficiency reaches the maximum value for different AFRs. Furthermore, as it can be known from Table 5, the net exergy efficiency under the eighteenth condition is the maximum, which means that the optimal operational parameters of the vertical cooler are $190 \mathrm{~kg} / \mathrm{s}$ for the AFR, and $353 \mathrm{~K}$ for the AIT. 


\section{Conclusions}

(1) A porous medium and local thermal non-equilibrium theories were adopted to establish a simulation model of the flow and heat transfer in a vertical cooler. The convective term with the particle movement was compiled into the simulation model through the transition of the source term in the solid energy equation on the basis of user-defined functions. The average deviation between the simulation values and the measurement values obtained from a self-made experimental cooler was $4.93 \%$, and the reliability and correctness of the established simulation model were verified.

(2) The SIT, AFR and AIT are three main operational parameters affecting the flow and heat transfer in a vertical cooler. Both the quantity of SWHR and energy efficiency increased as the AFR increased, and they decreased as the AIT increased. The increase in the SIT resulted in an increase in the quantity of SWHR, and a decrease in the energy efficiency.

(3) The air outlet exergy gradually increased as the SIT and AIT increased, while the air outlet exergy first increased and then decreased as the AFR increased. The increase in the SIT, AFR and AIT only led to an increase in the air pressure exergy. The change curve of the air net exergy could reach the maximum value as the AFR increased and gradually increased as the SIT and AIT increased. The increase in the SIT resulted in a decrease in the net exergy efficiency, and through the adjustment of the AFR and AIT, the vertical cooler can obtain the maximum net exergy efficiency.

(4) The AFR and AIT were determined as the optimized parameters, and the calculation method of the whole working condition was applied to obtain the optimal operational parameters of the vertical cooler by using the maximum net exergy efficiency. The actual sinter production of 3.9 million tons per year was taken as the processing capacity of the vertical cooler, and the optimal operational parameters of the vertical cooler were $190 \mathrm{~kg} / \mathrm{s}$ for the AFR, and $353 \mathrm{~K}$ for the AIT.

Author Contributions: All authors contributed to this research in collaboration. Z.C. and J.F. established the numerical calculation model and conducted the data management and analysis, H.W. and Y.X. proposed the analytical method of calculation data, and H.D. provided substantial help with the paper schedule. All authors have read and agreed to the published version of the manuscript.

Funding: The authors gratefully acknowledge the financial support for this work provided by the Anhui Provincial Key Research and Development Planning Foundation (201904a07020072, 202004a07020019, 202004a07020049), the National Natural Science Foundation of China (51974087), the Anhui Provincial Natural Science Foundation (1908085QE203) and the Anhui Jianzhu University Science Research Foundation (2020QDZ02).

Institutional Review Board Statement: Not applicable.

Informed Consent Statement: Not applicable.

Data Availability Statement: The data presented in this study are available on request from the corresponding author.

Conflicts of Interest: The authors declare no conflict of interest.

$\begin{array}{ll}\text { Abbreviations } & \\ 1 / a & \text { viscous resistance coefficient } \\ c & \text { specific heat }(\mathrm{J} /(\mathrm{kg} \cdot \mathrm{K})) \\ C_{2} & \text { inertial resistance coefficient } \\ D & \text { inner diameter of cooling section }(\mathrm{m}) \\ d_{\mathrm{p}} & \text { sinter equivalent diameter }(\mathrm{m}) \\ E x_{\mathrm{g}, \mathrm{out}} & \text { air outlet exergy }(\mathrm{MW}) \\ E x_{\mathrm{g}, \text { in }} & \text { air inlet exergy }(\mathrm{MW}) \\ E x_{\mathrm{g}, \mathrm{p}} & \text { air pressure exergy }(\mathrm{MW}) \\ E x_{\mathrm{g}, \text { net }} & \text { air net exergy (MW) }\end{array}$




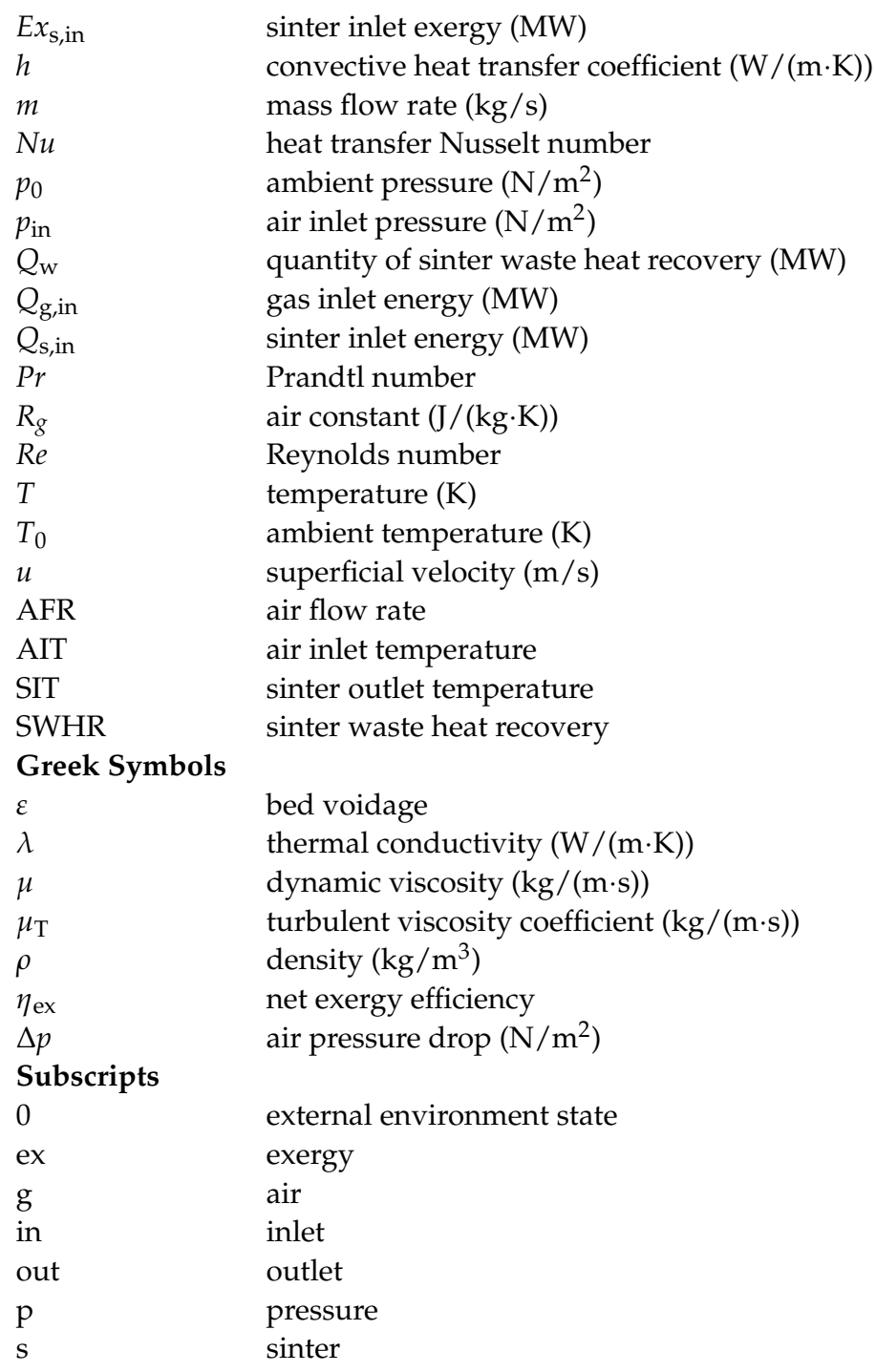

\section{References}

1. Dong, H.; Ling, H.Y.; Zhang, H.H.; Cai, J.J.; Xu, C.B.; Zhou, J.W. Thermal test and analysis of sintering cooling process. Iron Steel 2011, 46, 93-98.

2. Wang, X.D.; Tian, J.L.; Song, C.Y. Innovative practice technology and outlook in large iron and steel enterprise green manufacturing. Iron Steel 2018, 53, 1-9.

3. Cheng, Z.L.; Guo, Z.G.; Tan, Z.T.; Yang, J.; Wang, Q.W. Waste heat recovery from high-temperature solid granular materials: Energy challenges and opportunities. Renew. Sust. Energ. Rev. 2019, 116, 109428. [CrossRef]

4. Cheng, Z.L.; Tan, Z.T.; Guo, Z.G.; Yang, J.; Wang, Q.W. Recent progress in sustainable and energy-efficient technologies for sinter production in the iron and steel industry. Renew. Sust. Energ. Rev. 2020, 131, 110034. [CrossRef]

5. Dong, H.; Zhao, Y.; Cai, J.J.; Zhou, J.W.; Ma, G.Y. On the air leakage problem in sintering cooling system. Iron Steel 2012, 47, 95-99.

6. Cai, J.J.; Dong, H. Method and Device of Sinter Waste Heat Recovery and Utilization with Vertical Tank. Chinese Patent $200910187381.8,5$ January 2011.

7. Zhang, X.H.; Chen, Z.; Zhang, J.Y.; Ding, P.X.; Zhou, J.M. Simulation and optimization of waste heat recovery in sinter cooling process. Appl. Therm. Eng. 2013, 54, 7-15. [CrossRef]

8. Liu, Y.; Yang, J.; Cheng, Z.L.; Wang, J.; Wang, Q.W. Cost Benefits Analysis for Waste Heat Utilization in Sinter Cooling Bed. Chem. Eng. Trans. 2014, 39, 841-846.

9. Liu, Y.; Yang, J.; Wang, J.; Cheng, Z.L.; Wang, Q.W. Energy and exergy analysis for waste heat cascade utilization in sinter cooling bed. Energy 2014, 67, 370-380. [CrossRef]

10. Tian, W.Y.; Zhang, J.Y.; Dai, C.D.; Long, X.Y. Recovery of waste heat from sinter cooling process: Simulation and optimization. Ironmak. Steelmak. 2015, 42, 97-104. [CrossRef]

11. Feng, H.J.; Chen, L.G.; Liu, X.; Xie, Z.H.; Sun, F.R. Constructal optimization of a sinter cooling process based on exergy output maximization. Appl. Therm. Eng. 2016, 96, 161-166. [CrossRef] 
12. Zhang, S.; Feng, J.S.; Dong, H. Simulation and optimization of thermal parameter of sinter annular cooler based on enthalpy exergy of heat carrier. CIESC J. 2017, 68, 4129-4136.

13. Zhang, S.; Gao, J.Y.; Feng, J.S.; Dong, H. Simulation and optimization of heat recovery section of annular cooler based on enthalpy exergy of heat carrier. J. Cent. South Univ. (Sci. Technol.) 2018, 49, 2083-2090.

14. Zhang, S.; Zhao, L.; Feng, J.S.; Luo, X.F.; Dong, H. Parameter optimization of gas-solid heat transfer process in sinter packed bed based on further exergy analysis. Chem. Eng. Res. Des. 2019, 146, 499-508. [CrossRef]

15. Zhang, S.; Zhao, L.; Feng, J.S.; Dong, H. Effect of operating parameters on gas-solid exergy transfer performance in sinter annular cooler. Appl. Therm. Eng. 2020, 181, 115928. [CrossRef]

16. Tian, W.Y.; Jiang, C.; Ni, B.Y.; Wu, Z.T.; Wang, Q.; Yang, L.F. Global sensitivity analysis and multi-objective optimization design of temperature field of sinter cooler based on energy value. Appl. Therm. Eng. 2018, 143, 759-766. [CrossRef]

17. Tian, W.Y.; Ni, B.Y.; Jiang, C.; Wu, Z.T. Uncertainty analysis and optimization of sinter cooling process for waste heat recovery. Appl. Therm. Eng. 2019, 150, 111-120. [CrossRef]

18. Afridi, M.I.; Qasim, M.; Makinde, O.D. Entropy generation due to heat and mass transfer in a flow of dissipative elastic fluid through a porous medium. J. Heat Transfer. 2019, 141, 022002. [CrossRef]

19. Makinde, O.D.; Eegunjobi, A.S. Entropy analysis of a variable viscosity MHD couette flow between two concentric pipes with convective cooling. Eng. Trans. 2020, 68, 317-334.

20. Feng, J.S.; Dong, H.; Liu, J.Y.; Liang, K.; Gao, J.Y. Experimental study of gas flow characteristics in vertical tank for sinter waste heat recovery. Appl. Therm. Eng. 2015, 91,73-79. [CrossRef]

21. Feng, J.S.; Dong, H.; Gao, J.Y.; Liu, J.Y.; Liang, K. Exergy transfer characteristics of gas-solid heat transfer through sinter bed layer in vertical tank. Energy 2016, 111, 154-164. [CrossRef]

22. Feng, J.S.; Dong, H.; Gao, J.Y.; Liu, J.Y.; Liang, K. Theoretical and experimental investigation on vertical tank technology for sinter waste heat recovery. J. Cent. South Univ. 2017, 24, 2281-2287. [CrossRef]

23. Fu, Q.S. Thermodynamic Analysis Method of Energy System; Xi'an Jiaotong University Press: Xi'an, China, 2005.

24. Feng, J.S.; Dong, H.; Zhao, Y. Numerical investigation of gas flow in vertical tank for recovering sinter waste heat. J. Northeast. Univ. (Nat. Sci.) 2015, 36, 660-664.

25. Jang, J.Y.; Chiu, Y.W. 3-D Transient conjugated heat transfer and fluid flow analysis for the cooling process of sintered bed. Appl. Therm. Eng. 2009, 29, 2895-2903. [CrossRef]

26. Lesage, F.; Midoux, N.; Latifi, M.A. New local measurements of hydrodynamics in porous media. Exp. Fluid 2004, 37, 257-262. [CrossRef]

27. Yang, J.; Zeng, M.; Wang, Q.W.; Nakayama, A. Forced convection heat transfer enhancement by porous pin fins in rectangular channels. ASME J. Heat Transf. 2010, 132, 051702. [CrossRef]

28. Wakao, N.; Funazkri, T. Effect of fluid dispersion coefficients on particle-to-fluid mass transfer coefficients in packed beds. Chem. Eng. Sci. 1978, 33, 1375-1384. [CrossRef]

29. Feng, J.S. Study on Gas-Solid Heat Transfer Model in Vertical Tank for Recycling Sinter Waste Heat. Master's Thesis, Northeastern University, Shenyang, China, 2014.

30. Feng, J.S.; Dong, H.; Dong, H.D. Modification of Ergun's correlation in vertical tank for sinter waste heat recovery. Powder Technol. 2015, 280, 89-93. [CrossRef]

31. Kye, S.H.; Jae, H.J.; Won, K.L. Fixed-bed adsorption for bulk component system: Non-equilibrium non-isothermal and nonadiabatic model. Chem. Eng. Sci. 1995, 50, 813-825.

32. Feng, J.S.; Dong, H.; Gao, J.Y.; Liu, J.Y.; Liang, K. Experimental study of gas-solid overall heat transfer coefficient in vertical tank for sinter waste heat recovery. Appl. Therm. Eng. 2016, 95, 136-142. [CrossRef]

33. Su, J.W.; Gu, Z.L.; Chen, C.G.; Xu, X.Y. A two-layer mesh method for discrete element simulation of gas-particle systems with arbitrarily polyhedral mesh. Int. J. Num. Meth. Eng. 2015, 103, 759-780. [CrossRef]

34. Kamiński, M.; Carey, G.F. Stochastic perturbation-based finite element approach to fluid flow problems. Int. J. Numer. Methods H. 2005, 15, 671-697. [CrossRef]

35. Feng, J.S.; Dong, H.; Gao, J.Y.; Liu, J.Y.; Liang, K. Numerical investigation of gas-solid heat transfer process in vertical tank for sinter waste heat recovery. Appl. Therm. Eng. 2016, 107, 135-143. [CrossRef]

36. Tian, F.Y.; Huang, L.F.; Fan, L.W.; Weng, Y.K.; Ying, X.Y.; Yu, Z.T.; Cen, K.F. A comprehensive characterization on the structural and thermo-physical properties of sintered ore particles toward waste heat recovery applications. Appl. Therm. Eng. 2015, 90, 1007-1014. [CrossRef]

37. Feng, J.S.; Zhang, S.; Dong, H.; Pei, G. Effect of gas inlet parameters on exergy transfer performance of sinter cooling process in vertical moving bed. Appl. Therm. Eng. 2019, 152, 126-134. [CrossRef] 